This item was submitted to Loughborough's Research Repository by the author.

Items in Figshare are protected by copyright, with all rights reserved, unless otherwise indicated.

\title{
Object-oriented feature-based design
}

PLEASE CITE THE PUBLISHED VERSION

PUBLISHER

(C) Taylor \& Francis

VERSION

AM (Accepted Manuscript)

LICENCE

CC BY-NC-ND 4.0

REPOSITORY RECORD

Bin Wan Harun, Wan Abdul Rahman Jauhari, and Keith Case. 2019. "Object-oriented Feature-based Design". figshare. https://hdl.handle.net/2134/13527. 
This item was submitted to Loughborough's Institutional Repository (https://dspace.lboro.ac.uk/) by the author and is made available under the following Creative Commons Licence conditions.

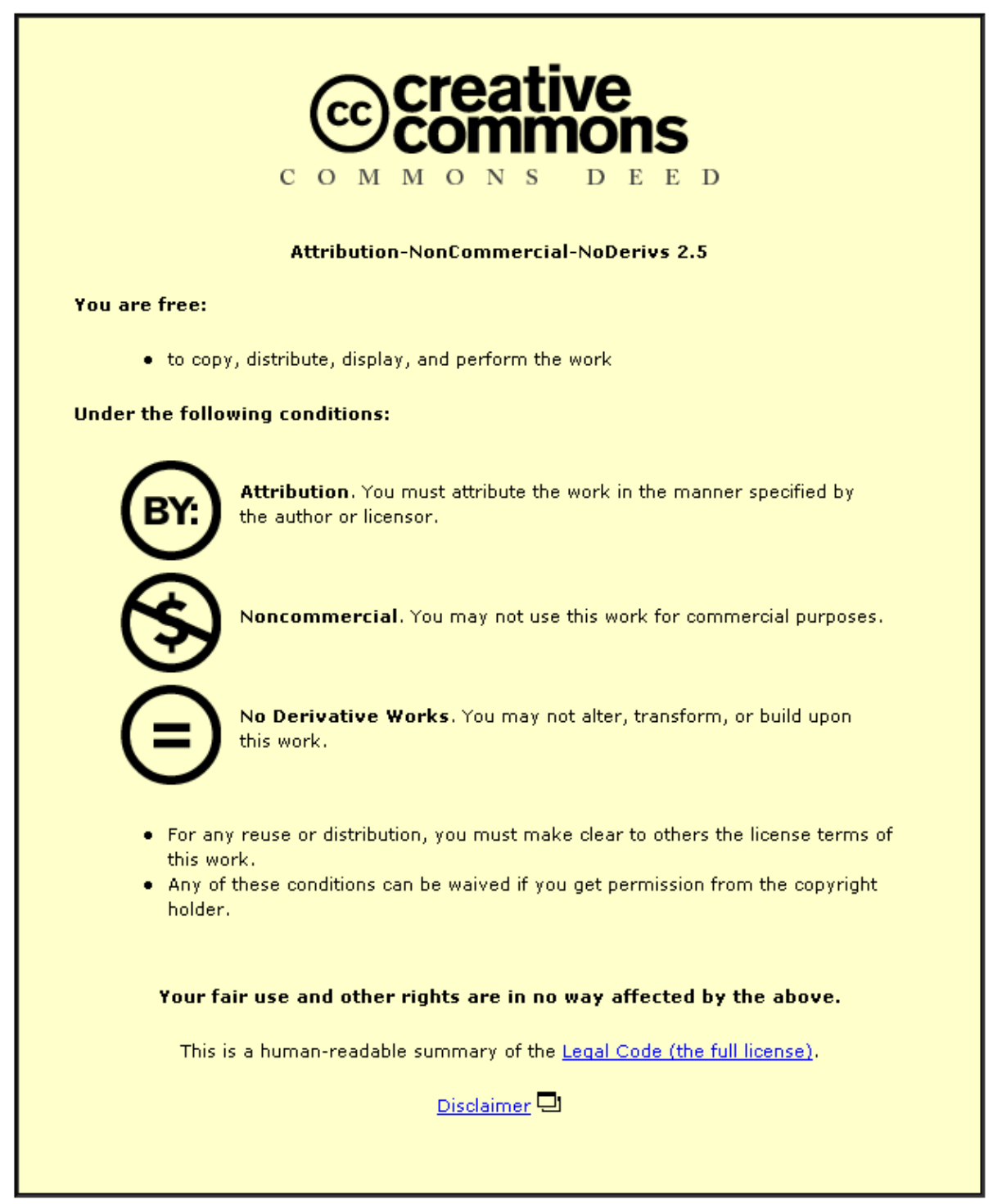

For the full text of this licence, please go to: http://creativecommons.org/licenses/by-nc-nd/2.5/ 
Wan Harun, W. A. R., \& Case, K. (1994).

Object-oriented feature-based design.

In K. Case, \& S. T. Newman (Eds.), 'Advances in Manufacturing Technology VIII', the Proceedings of the Tenth National Conference on Manufacturing Research, NCMR 1994 (pp. 294-298). Loughborough University of Technology, Loughborough, UK: London, UK, 5-7 September 1994: Taylor \& Francis.

\title{
OBJECT-ORIENTED FEATURE-BASED DESIGN
}

\author{
Wan Abdul Rahman Wan Harun and Dr Keith Case
}

Loughborough University of Technology, Loughborough, Leics, LE11 3TU

\begin{abstract}
The feature-based design system described in this paper is an enhancement of an earlier system which was implemented as part of an integrated design and process planning system. The feature taxonomy has been extended using an object-oriented approach to add functionality to the geometric reasoning process. This has been achieved by developing features within a library that is linked to the classes library provided by the ACIS ${ }^{\circledR}$ solid modeller. The explicit representation of potential assembly directions in the features make them suitable for assembly planning analysis. The research demonstrates the possibilities for a single feature representation to support multiple activities within a computer integrated manufacturing environment.
\end{abstract}

\section{Introduction}

The use of features is a prominent trend in research in the application of CAD/CAM systems in recent years. Feature technology is viewed as a key technology for the next generation of computer-aided design and manufacturing systems. Research in this area is aimed at determining alternative component representations which form a suitable basis for a wide ranging set of activities throughout a product's life cycle. Feature technology offers several advantages such as providing a more intuitive way of building up models and capturing more of the designer's intent in the product representation. Comprehensive reviews on feature- based design can be found in Salomons et. al. (1993) and (Case and Gao (1993).

This paper discusses the underlying methodology employed in the development of an extended version of a feature-based design system and its application in the assembly modelling environment. Earlier work related to research on process planning and process capability modelling for design and selection of processing equipment (Case 1994). The prototype system is fully integrated with a solid modeller kernel which enhances its 
modelling capabilities and provides an efficient tool to model a product. A feature-based library has been created and linked to the solid modeller library. The object-oriented approach is employed through the use of the $\mathrm{C}^{++}$programming language. The explicit representation of potential assembly directions in the current implementation of features means that the features are highly suited to the assembly planning activity. This is obtained through the information provided by the Boundary Representation (B-Rep) model represented by ACIS $®$.

\section{Feature Definition and Representation}

One of the requirements of a feature-based system is a feature representation that is capable of supporting many of the design and manufacturing planning activities.

Manufacturing planning systems need to extract feature-based component information from CAD systems both accurately and efficiently. In recent years, object-oriented techniques have been used widely in many computer applications. Object-oriented concepts are seen as fitting well within CAD framework by providing utilities for manipulating data, managing the permanent storage of data arid maintaining a user interface (Warman 1990). Using an object-oriented structure provides a general way to model and manipulate features for geometric reasoning (Unger and Ray 1988, Chung et. al. 1988). In this representation, features are conceived as objects that have data and a number of attributes to describe their characteristics. It is readily extensible to include additional data and relationships as appropriate. Examples of the object-oriented feature-based systems can be found in Unger and Ray (1988) and Chen et. al. (1994).

The feature taxonomy scheme used provides the required structure for feature primitives (Case et. al. 1994) and form features are treated as volumes enveloped by a set of real and imaginary faces. The real faces physically exist on the component and are typically surfaces from the original part or the result of manufacturing operations. Imaginary faces can be considered as the surfaces required together with the real faces to form an enclosed volume. Form features are divided into three categories - protrusions, depressions and surfaces. One of the characteristics of this feature taxonomy is the number of orthogonal directions from which the feature volume might be approached. These are known as External Access Directions (EAD's) and all features have between 0 and 6 EADs. Feature geometry is described by defining the EADs, the boundary type (open, closed) and the exit boundary status (through/not through). Compound features and parent-child relationships among features have also been defined.

The above classification of features provides a convenient method for representation using an object oriented approach. Two main base classes are created-features and profiles, based on the feature definition and taxonomy described above. The feature class defines data such as feature identities, feature type and surface roughness. For each feature class, a number of primitive shapes are defined, boss, pocket, hole, non-through slot, through slot, notch, step and real face, as shown in Figure1. Each feature type class has its own data such as the number of EADs, its dimensions, location and orientation with respect to the component. Procedures are also available for feature operators, such as create, edit, move, 
copy and rotate features. Further procedures determine mating surfaces, define parent-child relationships and the topological data for assembly planning. The modular nature of the object-oriented technique permits feature classes to be extended to allow other procedures to be added in the future.

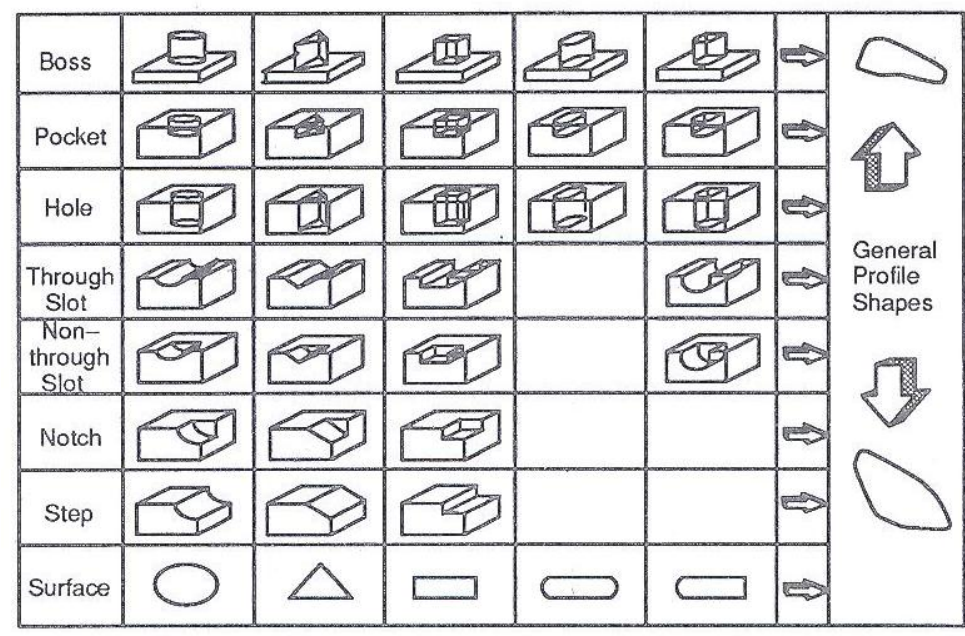

Figure 1 . The library of feature primitives

The profile class is a base class for standard profile shapes common to each type of feature, such as circular, rectangular and triangular as well as a user defined profile. For example, a boss may have a circular profile and a pocket a rectangular shape. Other profiles can be defined by the user by entering the appropriate parameters. The primitive shapes of all feature classes form the feature library from which feature instances can be generated by retrieving the feature primitives and specifying the parameters. The two classes are referenced by each other. For instance, a boss would reference a profile object, where this profile object is actually an object of a derived class of profile. The relationships among classes are shown in Figure 2.

\section{The User Interface System}

The system utilises the openness of the ACIS kernel modeller, which serves as the main engine of the system. ACIS is an object-oriented geometric modelling toolkit designed for use as a geometry engine within 3-D modelling applications (Spatial 1993). The modular architecture of ACIS provides extensive facilities for the development of a feature-based system. It also provides a rich set of geometric operators for the construction and manipulation of complex models.

The solid model is built within ACIS using class libraries which are accessed through an Application Procedural Interface (API) or direct-object interface to all internal objects, classes and methods. Some of the functions in the feature modelling system such as blending, sweeping and Boolean operations are derived or extended from these classes. The direct interface method allows the topology to be scanned to the face level while at the same 


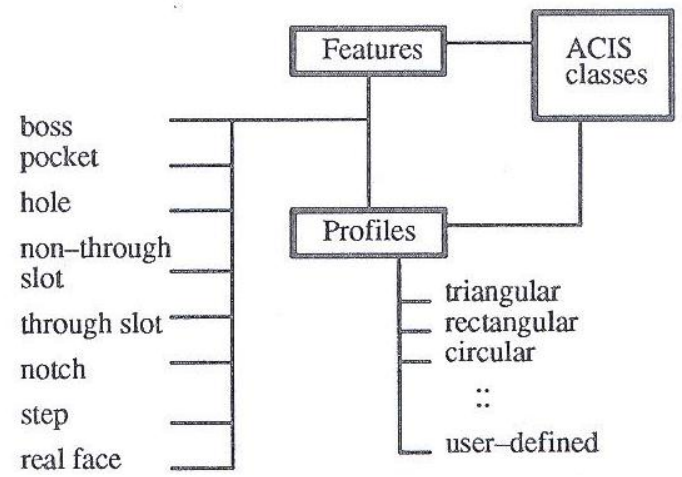

Figure 2. Class relationship

time the geometry is interrogated. ACIS also provides a mechanism to attach classes for assembly modelling attributes to any object in the data structure. Attributes are crucial for extending geometry models to become true product models. This includes complex attributes, which may represent dimensions, constraints on features and references to other objects.

A design by feature user interface to the system has been created to allow designers to generate components using feature primitives and to store attributes in a feature based data structure which is separate from, but associated with the database of the geometric modeller. The design by features interface allows designers to create features by defining the sets of parameters for feature primitives; to perform feature edit operations such as move, rotate, copy and delete: and to define feature relationships such as parent-child relationships and tolerances between features. The user interface to the system is provided by Motif. The system architecture is shown in Figure 3.

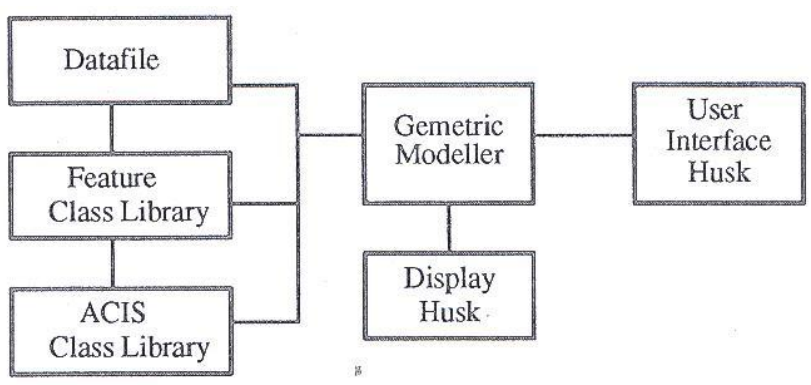

Figure 3. System architecture

A feature primitive is defined as the combination of a set of functions. For example, the create pocket function is the combination of create wire profile, cover the faces and sweep the faces. Once a feature is created, a B-Rep model is generated for the feature. All data on the model and features can be stored in a data file provided by the ACIS bulletin 
board. The latter contains data structure information recorded as a result of operational changes made to the features and can be accessed by the user for manipulation.

\section{Modelling Assemblies with Features}

Modelling assemblies is a way of examining complex geometric interactions before anything is built. Its aim is to describe the geometry and to define the relations between parts of the final assembly. Analysis and modelling of the final product constitute one of the most important steps in assembly planning. The product model must contain the data required to generate the assembly sequences. The explicit representation of assembly directions in the features is provided by the EADs defined in each feature. The position and the orientation of the feature, which can be accessed through functions provided by ACIS, can determine the location of the mating faces and the interactions of various features in the assembly. The work on this aspect is still under investigation.

\section{Conclusions}

The object-oriented approach described above is an effective way to implement geometric modelling in a feature-based system. In general, the modular nature of the objectoriented technique allows modelling at a higher level of complexity than is possible in current systems. It also enables easier extension to the modelling system. The application of the system for the assembly modelling is used to verify the generic nature of the approach.

\section{References}

Case, K. and Gao, J. 1993, Feature technology- an overview, Int Journal Computer Integrated Manufacturing, 6, Nos 1 \& 2, 2-12.

Case, K. 1994, Using a design by features CAD system for process capability modelling, Computer Integrated Manufacturing Systems, 7 (1), 39-49.

Case, K., Gao, J. X. and Gindy, N. N. Z. 1994, The implementation of a feature-based component representation for CAD/CAM integration, Proc Institution Mech Engrs. (IMechE), 208, 71-79.

Chen, C., Swift, F., Lee, S., Ege, R. and Shen, Q. 1994, Development of a feature-based and object-oriented concurrent engineering system, Journal of Intelligent Manufacturing, 5, 23 31.

Chung, J.C.H. 1988, Feature-based geometry construction for geometric reasoning, Proc ASME Computers in Engineering Conf. San Francisco, 497-504.

Salomons, O.W., van Houten, F.J.A.M. and Kals, H.J .J. 1993, Review of research in feature-based design, Journal of Manufacturing Systems, 12 (2), 113- 132.

Spatial Technology Inc. 1993, ACIS Geometric Modeler, Programmers Manual.

Unger, M.B. and Ray, S.R. 1988, Feature-based process planning in the AMRF, Proc ASME Computers in Engineering Conf, San Francisco, 563-569.

Warman, E. A. 1990, Object-oriented programming and CAD, Journal of Engineering

Design, 1, No 1, 37-46. 